Pacific

Journal of

Mathematics

A SHARP DIVISION ESTIMATE FOR ULTRADIFFERENTIABLE GERMS

VinCENT ThILLIEZ 


\title{
A SHARP DIVISION ESTIMATE FOR ULTRADIFFERENTIABLE GERMS
}

\author{
VINCENT THILLIEZ
}

Let $f$ (resp. $\varphi$ ) be a $C^{\infty}$ (resp. real-analytic) function germ near the origin in $\mathrm{R}^{n}$. Assume that $f$ is divisible by $\varphi$ in $C^{\infty}$, and that it belongs to a sufficiently regular utradifferentiable class $\left\{\ell ! M_{\ell}\right\}$ of Carleman type (for example, one of the Gevrey rings familiar in the theory of differential equations). What can then be said about the regularity of the quotient $f / \varphi$ ? In this paper, we obtain first a complete solution of this problem in the case $n=2$. Namely, it is shown that $f / \varphi$ belongs to the Carleman class $\left\{\ell ! M_{\ell}^{d(\varphi)}\right\}$, where $d(\varphi)$ is a suitable Łojasiewicz exponent for the regular separation between the space $\mathbf{R}^{2}$ and certain components of the complex zero set $Z_{\varphi}$ of $\varphi$. This number can be explicitely computed by means of Puiseux expansions. We prove moreover that the division result is sharp for any $\varphi$ and $M$. Finally, we apply it to get a characterization of closed principal ideals generated by real-analytic functions in Carleman classes of two variables, improving a result which was known previously only in the case of generators with isolated real zeros.

\section{Introduction.}

Many studies in differential analysis involve a more or less explicit version of the following division problem: Let $\varphi$ be a given real-analytic function germ at the origin in $\mathbf{R}^{n}$ and let $f$ be another germ enjoying some regularity properties. What kind of control on the quotient $f / \varphi$ is it then possible to derive from the knowledge of geometric and differential properties of $\varphi$ and $f$ ? As typical examples of questions in this spirit, we refer the reader to the Łojasiewicz division theorem [10], [11], the work of MostowShnider on continuity of $C^{\infty}$ quotients [12], theorems of Izumi [8] and Wang [17] on Chevalley estimates, and so on. Of course, the kind of regularity property which is dealt with (order of vanishing, smoothness, estimates on derivatives...) has a strong influence on the nature of the corresponding result.

The present article studies division estimates in the setting of very smooth germs, namely ultradifferentiable classes. Denote by $C^{\infty}\left(\mathbf{R}^{n}, 0\right)$ the ring of infinitely differentiable function-germs at the origin in $\mathbf{R}^{n}$, and recall 
that the Carleman class of germs $C_{M}\left(\mathbf{R}^{n}, 0\right)$ is defined as the subclass of $C^{\infty}\left(\mathbf{R}^{n}, 0\right)$ containing all those germs $f$ whose derivatives at any order $\ell$ are majorized by $C_{f}^{\ell+1} \ell ! M_{\ell}$ in some fixed neighborhood of 0 , where $C_{f}$ is a constant depending on $f$, and $\left(M_{\ell}\right)_{\ell \geq 0}$ is a given sequence of real numbers satisfying suitable regularity assumptions. In some sense, the role of this sequence consists in putting a bound on the defect of analyticity of the elements of $C_{M}\left(\mathbf{R}^{n}, 0\right)$. Thus, such classes provide scales of regularity between the analytic and the $C^{\infty}$ ones. A well-known and much studied example is given by the scale of Gevrey classes $\left(M_{\ell}=\ell !^{\alpha}\right.$ with $\left.\alpha>0\right)$. In Carleman classes, the general division problem described above can be precisely stated as follows: Being given a germ $\varphi$ of real-analytic function at the origin in $\mathbf{R}^{n}$ and a Carleman class $C_{M}\left(\mathbf{R}^{n}, 0\right)$, investigate the smallest possible class $C_{M^{+}}\left(\mathbf{R}^{n}, 0\right)$ containing $C_{M}\left(\mathbf{R}^{n}, 0\right)$ and enjoying the following property: If $f$ is an element of $C_{M}\left(\mathbf{R}^{n}, 0\right)$ which can be written as $\varphi g$ for some $g$ belonging to $C^{\infty}\left(\mathbf{R}^{n}, 0\right)$, then the quotient $g=f / \varphi$ belongs to $C_{M^{+}}\left(\mathbf{R}^{n}, 0\right)$. Of course, the sequence $M^{+}$should depend only on $M$ and on geometric and differential properties of $\varphi$. The reason why we consider different classes $C_{M}$ and $C_{M^{+}}$lies in the fact that some loss of regularity turns out to be generally unavoidable in the division process; hence the class of the quotient has to be (possibly strictly) wider than $C_{M}$. The only exception arises for $n=1$, where obvious arguments show that $M^{+}=M$.

In this paper, we obtain a complete solution of the problem for $n=2$, that is, for germs in two variables. As a starting point, we mention that in the particular case where the real zero set of $\varphi$ reduces to the single point $\{0\}$, it is possible to see, as a corollary of the results in [15], that the solution is given by $M_{\ell}^{+}=M_{\ell}^{\tau(\varphi)}$, where $\tau(\varphi)$ is the Eojasiewicz exponent for the regular separation between $\mathbf{R}^{2}$ and the complex zero set of $\varphi$. Moreover, this choice of $M^{+}$is sharp. However, after [15], the previous division problem remained unsolved for general zero sets, even for two variables. In this setting, we shall show here (Theorem 2.1) that the previous solution is still valid, provided we extend the definition of $M^{+}$by putting

$$
M_{\ell}^{+}=M_{\ell}^{d(\varphi)},
$$

where $d(\varphi)$ is, roughly speaking, the greatest number among all Łojasiewicz exponents for the regular separation between $\mathbf{R}^{2}$ and one of those branches of the complex zero set of $\varphi$ whose intersection with a half-plane in $\mathbf{R}^{2}$ reduces to $\{0\}$ (see Section 1 for details). Of course, we have $d(\varphi)=\tau(\varphi)$ when the intersection of the zero set itself with $\mathbf{R}^{2}$ reduces to $\{0\}$.

Our method of proof relies exclusively on explicit estimates, striving to stay as accurate as possible at each step. The underlying reason why dimension 2 plays a special role lies in the fact that, as in [15], it allows to relate $d(\varphi)$ to some numbers occuring explicitely in Puiseux expansions of 
the roots of $\varphi$. However, some particular results can be extended to the higher dimensional case; see the details in Subsection 4.3 below. We owe these extensions to Edward Bierstone and we take here the opportunity to thank him for his insightful comments on the subject.

Finally, we mention that another strategy to study such division problems in certain classes of smooth functions has been developed by Bos-Milman [4] and Chaumat-Chollet [7]: Using resolution of singularities, these authors are led to a quite different kind of results, as explained in Remark 4.4 below.

The article is organized as follows: Section 1 recalls definitions and various prerequisites; Section 2 states and partly proves the main result (Theorem 2.1). Section 3 is devoted to establishing a technical estimate, thus completing the proof of Theorem 2.1; this is the most delicate part of the paper. In Section 4, we discuss the sharpness of the main result (see Theorem 4.1); we apply it to a characterization of closed ideals (Theorem 4.2) and give some comments.

\section{Preliminaries.}

1.1. Notations. Throughout the paper, for any multi-index $L=\left(\ell_{1}, \ldots\right.$, $\ell_{n}$ ) in $\mathbf{N}^{n}$, we shall denote by the corresponding lower case letter $\ell$ the length $\ell_{1}+\cdots+\ell_{n}$ of $L$. We also put $L !=\ell_{1} ! \ldots \ell_{n}$ ! and denote by $D^{L}$ the operator $\partial_{1}^{\ell_{1}} \ldots \partial_{n}^{\ell_{n}}$, with $\partial_{j}=\partial / \partial x_{j}$ for $j=1, \ldots, n$. We say that a $C^{\infty}$ germ $f$ is flat at a point $a$ if $f$ and all its derivatives vanish at $a$. For any subset $E$ of $\mathbf{R}^{n}$ or $\mathbf{C}^{n}$, we shall sometimes write $(E, 0)$ to denote $E \cap U$ where $U$ is some neighborhood of 0 . When we write that a property $\mathcal{P}(x, y)$, depending on $x \in \mathbf{R}^{n}$ and on some family of other parameters $y$, holds for $x$ in $(E, 0)$, it is assumed that the neighborhood $U$ does not depend on $y$. We denote by $d(x, E)$ the euclidean distance from a point $x$ to $E$. At last, the following classical notation will be useful: If $A(t)$ and $B(t)$ are two positive functions of a variable $t$ belonging to a set $T$, one writes $A(t) \lesssim B(t)$ (or equivalently $B(t) \gtrsim A(t))$ to say that there exists a real $C$, not depending on $t$, such that the inequality $A(t) \leq C B(t)$ holds for all $t \in T$. The notation $A(t) \approx B(t)$ means that one has $A(t) \lesssim B(t)$ and $B(t) \lesssim A(t)$ simultaneously.

1.2. On ultradifferentiable germs. Let $M=\left(M_{\ell}\right)_{\ell \geq 0}$ be an increasing sequence of real numbers with $M_{0}=1$. The sequence $M$ is said to be strongly regular if there exists a positive constant $A$ such that the following assumptions are satisfied:

$M$ is logarithmically convex,

$$
\begin{gathered}
M_{j+k} \leq A^{j+k} M_{j} M_{k} \text { for any }(j, k) \in \mathbf{N}^{2}, \\
\sum_{j \geq \ell} \frac{M_{j}}{(j+1) M_{j+1}} \leq A \frac{M_{\ell}}{M_{\ell+1}} \text { for any } \ell \in \mathbf{N} .
\end{gathered}
$$


Condition (1), which amounts to saying that $M_{\ell+1} / M_{\ell}$ increases with $\ell$, implies

$$
M_{j} M_{k} \leq M_{j+k} \text { for any }(j, k) \in \mathbf{N}^{2} .
$$

Thus (2), which is in some sense converse to (4), means that the growth of $M$ is not too wild. The role of condition (3) will be recalled later, after the definition of ultradifferentiable germs. To each strongly regular sequence $M$, one associates classically the function $h_{M}$ defined by $h_{M}(t)=$ $\inf _{\ell \geq 0} t^{\ell} M_{\ell}$ for any $t \in \mathbf{R}_{+}$. This function, which is a sort of Legendre transform, is continuous, non-decreasing; we have $h_{M}(0)=0$ and $h_{M}(t)=1$ for $t \geq 1$. In virtue of (1), the knowledge of $h_{M}$ fully determines the sequence $M$ since we have

$$
M_{\ell}=\sup _{t>0} t^{-\ell} h_{M}(t) \text { for any } \ell \in \mathbf{N} .
$$

For the typical example of Gevrey sequences $M_{\ell}=\ell !^{\alpha}(\alpha>0)$, we have $h_{M}(t)=\exp (-\lambda(t))$ with $\lambda(t) \approx t^{-1 / \alpha}$.

Now let $s$ be a real number with $s \geq 1$. Obviously, the sequence $M^{s}=$ $\left(M_{\ell}^{s}\right)_{\ell \geq 0}$ is also strongly regular, and we have $h_{M^{s}}\left(t^{s}\right)=\left(h_{M}(t)\right)^{s}$. We recall also that there exists a constant $\rho(s) \geq 1$, depending only on $s$ and $M$, such that

$$
h_{M}(t) \leq\left(h_{M}(\rho(s) t)\right)^{s} \text { for any } t \in \mathbf{R}_{+} .
$$

From (2), (5) and (6), it is straightforward to deduce that there exists a positive constant $A(s)$, depending only on $s$ and $M$, such that

$$
t^{-s \ell} h_{M}(t) \leq A(s)^{\ell} M_{\ell}^{s} h_{M}(A(s) t) \text { for any } t \in \mathbf{R}_{+}^{*} \text { and } \ell \in \mathbf{N} .
$$

As in [15], for any strongly regular sequence $M$, we define now $C_{M}\left(\mathbf{R}^{n}, 0\right)$ as the class of germs $f$ in $C^{\infty}\left(\mathbf{R}^{n}, 0\right)$ for which there exist a neighborhood $U$ of 0 in $\mathbf{R}^{n}$ and a constant $C>0$ such that the inequality

$$
\left|D^{L} f(x)\right| \leq C^{\ell+1} \ell ! M_{\ell}
$$

holds for any $x$ in $U$ and any multi-index $L$ in $\mathbf{N}^{n}$. The class $C_{M}\left(\mathbf{R}^{n}, 0\right)$ is a local algebra, stable under derivation. As remarked in the introduction, the sequence $M$ majorizes, in some sense, the defect of analyticity of its elements. Condition (3) is the so-called strong non-quasianalyticity condition ensuring the existence of "good" cutoff functions with $C_{M}$ regularity [5]. For a more detailed interpretation of (1), (2), (3) in terms of ultradifferentiable classes, we refer the reader to $[\mathbf{5}],[\mathbf{9}],[\mathbf{1 3}]$. Beware that the notations in $[\mathbf{5}],[\mathbf{9}],[\mathbf{1 3}]$ are different, since $M_{\ell}$ plays there the role played by $\ell ! M_{\ell}$ in the present paper. The latter notation, viewing $M$ as a defect of analyticity, is more convenient here, since it seems, as a general rule, that any loss of regularity involved by the various operations of differential analysis (division, etc.) in the setting of ultradifferentiable functions, acts only on the defect of analyticity. 
1.3. On analytic germs. Denote by $\mathcal{O}\left(\mathbf{R}^{n}, 0\right)\left(\operatorname{resp} . \mathcal{O}\left(\mathbf{C}^{n}, 0\right)\right)$ the ring of real-analytic (resp. holomorphic) function germs at the origin in $\mathbf{R}^{n}$ $\left(\right.$ resp. $\left.\mathbf{C}^{n}\right)$. By means of the power series expansion, $\mathcal{O}\left(\mathbf{R}^{n}, 0\right)$ can obviously be viewed as the subset of elements of $\mathcal{O}\left(\mathbf{C}^{n}, 0\right)$ taking real values on $\mathbf{R}^{n}$. Thus, to each real-analytic germ $\gamma$ in $\mathcal{O}\left(\mathbf{R}^{n}, 0\right)$, one can associate the set of its complex zeros $Z_{\gamma}=\gamma^{-1}(0)$ as a germ of complex analytic set.

From now on, we shall denote by $\omega(v)$ the order of any element $v$ of $\mathcal{O}(\mathbf{C}, 0)$, that is the least degree of monomials occuring in the power series expansion of $v$, with the convention $\omega(0)=\infty$.

Let $\varphi$ be a given element of $\mathcal{O}\left(\mathbf{R}^{2}, 0\right)$. After a linear change of coordinates with real matrix, one can clearly assume, without changing the fact that $\varphi$ is real, that the $z_{1}$-axis is transversal to the zero set $Z_{\varphi}$ at 0 . Using Puiseux's theorem as in [15], we can write, for some integer $m$ in $\mathbf{N}^{*}$,

$$
\varphi\left(z_{1}, z_{2}^{m}\right)=u\left(z_{1}, z_{2}^{m}\right) \prod_{p=1}^{p_{1}}\left(z_{1}-U_{p}\left(z_{2}\right)\right)^{n_{p}}
$$

where $n_{1}, \ldots, n_{p_{1}}$ are positive integers, $u$ is a unit in $\mathcal{O}\left(\mathbf{C}^{2}, 0\right)$ and $U_{1}, \ldots$, $U_{p_{1}}$ is a suitable family of germs in $\mathcal{O}(\mathbf{C}, 0)$, satisfying $\omega\left(U_{p}\right) \geq m$ for $p=$ $1, \ldots, p_{1}$. Put $U_{p}=R_{p}+i S_{p}$ with $R_{p} \in \mathcal{O}(\mathbf{R}, 0)$ and $S_{p} \in \mathcal{O}(\mathbf{R}, 0)$. Using the fact that $\varphi$ is real, it is not difficult to see that for $x_{2}$ in $\mathbf{R}^{*}$, the roots $U_{p}\left(x_{2}\right)$ for which $S_{p}\left(x_{2}\right)$ is non-identically zero are pairwise conjugate. After reindexation, we can thus write, for some suitable integer $p_{0}$ satisfying $2 p_{0} \leq$ $p_{1}$,

$$
U_{p}= \begin{cases}R_{p}+i S_{p} & \text { with } m \leq \omega\left(S_{p}\right)<\infty \text { for } p=1, \ldots, p_{0} \\ R_{p-p_{0}}-i S_{p-p_{0}} & \text { for } p=p_{0}+1, \ldots, 2 p_{0} \\ R_{p} & \text { with } \omega\left(R_{p}\right) \geq m \text { for } p=2 p_{0}+1, \ldots, p_{1} .\end{cases}
$$

Note that we can write

$$
\varphi\left(z_{1}, z_{2}^{m}\right)=u\left(z_{1}, z_{2}^{m}\right) \prod_{p=1}^{p_{0}}\left(\psi_{p}\left(z_{1}, z_{2}\right)\right)^{n_{p}} \prod_{p=2 p_{0}+1}^{p_{1}}\left(z_{1}-R_{p}\left(z_{2}\right)\right)^{n_{p}}
$$

with $\psi_{p}\left(z_{1}, z_{2}\right)=\left(z_{1}-R_{p}\left(z_{2}\right)\right)^{2}+\left(S_{p}\left(z_{2}\right)\right)^{2}$; and we have also $u \in \mathcal{O}\left(\mathbf{R}^{2}, 0\right)$ since $\varphi$ is real. The special case $p_{1}=2 p_{0}$, occuring when $Z_{\varphi} \cap\left(\mathbf{R} \times \mathbf{R}_{+}\right)=$ $\{0\}$, has been studied in detail in [15]. In the same spirit, we define here

$$
\begin{aligned}
& \mu_{p}=\omega\left(S_{p}\right) \text { and } \tau_{p}^{+}=\mu_{p} / m \text { for } p=1, \ldots, p_{0}, \\
& d^{+}(\varphi)=\max \left\{\tau_{p}^{+} ; p=1, \ldots, p_{0}\right\} \text { if } p_{0} \geq 1 \text { and } d^{+}(\varphi)=1 \text { if } p_{0}=0, \\
& d^{-}(\varphi)=d^{+}(\check{\varphi}) \text { with } \check{\varphi}\left(z_{1}, z_{2}\right)=\varphi\left(z_{1},-z_{2}\right) \\
& d(\varphi)=\max \left(d^{+}(\varphi), d^{-}(\varphi)\right) .
\end{aligned}
$$


We denote by $X_{\varphi}$ the set $Z_{\varphi} \cap \mathbf{R}^{2}$ of real zeros of $\varphi$. For technical reasons, we also define, for $p=1, \ldots, p_{1}$,

$$
\begin{aligned}
& Z_{p}=\left\{z ; \operatorname{Re} z_{2} \geq 0 \text { and } z_{1}=U_{p}\left(z_{2}^{1 / m}\right)\right\}, \quad X_{p}=Z_{p} \cap \mathbf{R}^{2}, \\
& Z_{\varphi}^{ \pm}=\left\{z \in Z_{\varphi} ; \operatorname{Re} z_{2} \in \mathbf{R}_{ \pm}\right\}, \quad X_{\varphi}^{ \pm}=Z_{\varphi}^{ \pm} \cap \mathbf{R}^{2},
\end{aligned}
$$

where $z_{2}^{1 / m}$ is the principal determination of the $m^{\text {th }}$-root of $z_{2}$.

1.4. On Łojasiewicz exponents. Let $\mathcal{X}$ and $\mathcal{Y}$ be two germs of closed subsets at the origin in $\mathbf{R}^{n}$, with $0 \in \mathcal{X} \cap \mathcal{Y}$; and let $\mathcal{T}$ be the set of real numbers $t \geq 1$ for which there exists a constant $c_{t}>0$ such that one has

$$
d(x, \mathcal{Y}) \geq c_{t} d(x, \mathcal{X} \cap \mathcal{Y})^{t} \text { for any } x \in(\mathcal{X}, 0) .
$$

Recall that $\mathcal{X}$ and $\mathcal{Y}$ are said to be regularly separated if $\mathcal{T}$ is not empty (it is classical that this definition is symmetric with respect to $\mathcal{X}$ and $\mathcal{Y}$, see e.g., [11]). The Eojasiewicz exponent for the regular separation between $\mathcal{X}$ and $\mathcal{Y}$ is then defined as the infimum of $\mathcal{T}$. As a fundamental fact of subanalytic geometry, it is known that the subanalyticity of $\mathcal{X}$ and $\mathcal{Y}$ implies regular separation, and that the Łojasiewicz exponent is rational and attained (in other words, it belongs to $\mathcal{T}$ ), see [3]. If it is equal to 1 , the sets $\mathcal{X}$ and $\mathcal{Y}$ are said to intersect transversally.

Here, according to [15], the number $d^{+}(\varphi)$ defined in the previous subsection is precisely the Łojasiewicz exponent for the regular separation between $\mathbf{R}^{2}$ and the union $\bigcup_{1 \leq p \leq 2 p_{0}} Z_{p}$ of all those "branches" $Z_{p}$ in $Z_{\varphi}^{+}$for which $X_{p}=\{0\}$ (for the other ones, $X_{p}$ is a $C^{1}$ real arc originating in $\{0\}$, and $Z_{p}$ intersects $\mathbf{R}^{2}$ transversally, as shown in Lemma 2.3 below). Notice also that when we have $Z_{\varphi} \cap \mathbf{R}^{2} \neq\{0\}$, the number $d(\varphi)$, which has been defined as $\max \left(d^{+}(\varphi), d^{-}(\varphi)\right)$, generally does not coincide anymore with the Lojasiewicz exponent for the regular separation between $\mathbf{R}^{2}$ and $Z_{\varphi}$. Indeed, it is possible to check that the latter exponent $\tau(\varphi)$ always satisfies $\tau(\varphi) \leq d(\varphi)$; but this inequality can be strict, see the following Example (iv).

\subsection{Examples.}

(i) $\varphi(x)=x_{1}^{2}-x_{2}^{2 k}\left(k \in \mathbf{N}^{*}\right)$. This the simplest kind of example: We can write $\varphi(x)=\check{\varphi}(x)=\left(x_{1}-x_{2}^{k}\right)\left(x_{1}+x_{2}^{k}\right)$, hence $m=1, p_{1}=2, p_{0}=0$ for both $\varphi$ and $\check{\varphi}$. We derive $d^{+}(\varphi)=d^{-}(\varphi)=d(\varphi)=1$.

(ii) $\varphi(x)=x_{1}^{2}-x_{2}^{2 k+1}\left(k \in \mathbf{N}^{*}\right)$, for which $X_{\varphi}$ is a cusp. We can write $\varphi\left(x_{1}, x_{2}^{2}\right)=\left(x_{1}-x_{2}^{2 k+1}\right)\left(x_{1}+x_{2}^{2 k+1}\right)$, hence $m=2, p_{1}=2, p_{0}=0$ and $d^{+}(\varphi)=1$. On the other hand, we have $\check{\varphi}(x)=x_{1}^{2}+x_{2}^{2 k+1}$, thus $\check{\varphi}\left(x_{1}, x_{2}^{2}\right)=\left(x_{1}-i x_{2}^{2 k+1}\right)\left(x_{1}+i x_{2}^{2 k+1}\right)$ : For this one, we get $m=2$, $p_{1}=2, p_{0}=1, \mu_{1}=2 k+1$, hence $d^{-}(\varphi)=d^{+}(\check{\varphi})=k+\frac{1}{2}$. We derive finally $d(\varphi)=k+\frac{1}{2}$. 
(iii) $\varphi(x)=\left(x_{1}^{2}-x_{2}^{3}\right)\left(x_{1}^{3}-x_{2}^{4}\right)$. We have

$$
\begin{aligned}
& \varphi\left(x_{1}, x_{2}^{6}\right)= \\
& \left(x_{1}+\frac{1}{2} x_{2}^{8}+i \frac{\sqrt{3}}{2} x_{2}^{8}\right)\left(x_{1}+\frac{1}{2} x_{2}^{8}-i \frac{\sqrt{3}}{2} x_{2}^{8}\right)\left(x_{1}-x_{2}^{8}\right)\left(x_{1}-x_{2}^{9}\right)\left(x_{1}+x_{2}^{9}\right) .
\end{aligned}
$$

This yields $m=6, p_{1}=5, p_{0}=1, \mu_{1}=8$ and consequently $d^{+}(\varphi)=$ $4 / 3$. We have also $\check{\varphi}(x)=\left(x_{1}^{2}+x_{2}^{3}\right)\left(x_{1}^{3}-x_{2}^{4}\right)$, hence

$$
\begin{aligned}
& \check{\varphi}\left(x_{1}, x_{2}^{6}\right)= \\
& \left(x_{1}-i x_{2}^{9}\right)\left(x_{1}+i x_{2}^{9}\right)\left(x_{1}+\frac{1}{2} x_{2}^{8}+i \frac{\sqrt{3}}{2} x_{2}^{8}\right)\left(x_{1}+\frac{1}{2} x_{2}^{8}-i \frac{\sqrt{3}}{2} x_{2}^{8}\right)\left(x_{1}-x_{2}^{8}\right) .
\end{aligned}
$$

For $\check{\varphi}$, we can thus take $m=6, p_{1}=5, p_{0}=2, \mu_{1}=9, \mu_{2}=8$ and $d^{-}(\varphi)=d^{+}(\check{\varphi})=3 / 2$. Finally, we get $d(\varphi)=3 / 2$.

(iv) $\varphi(x)=x_{1}\left(\left(x_{1}-x_{2}^{2}\right)^{2}+x_{2}^{8}\right)$. We can write

$$
\varphi(x)=\check{\varphi}(x)=x_{1}\left(x_{1}-x_{2}^{2}+i x_{2}^{4}\right)\left(x_{1}-x_{2}^{2}-i x_{2}^{4}\right),
$$

hence $m=1, p_{1}=3, p_{0}=1, \mu_{1}=4$ and $d(\varphi)=4$. Now we compute the Eojasiewicz exponent $\tau(\varphi)$ for the regular separation between $\mathbf{R}^{2}$ and $Z_{\varphi}$. In $\mathbf{C}^{3}$, the set $Z_{\varphi}$ has 3 smooth irreducible components $Z^{\prime}, Z_{+}^{\prime \prime}$, $Z_{-}^{\prime \prime}$ respectively given by $Z^{\prime}=\left\{z_{1}=0\right\}$ and $Z_{ \pm}^{\prime \prime}=\left\{z_{1}-z_{2}^{2} \pm i z_{2}^{4}=0\right\}$. Pick $x \in\left(\mathbf{R}^{2}, 0\right)$. Elementary arguments show that $d\left(x, Z^{\prime}\right)=\left|x_{1}\right|$ and $d\left(x, Z_{ \pm}^{\prime \prime}\right) \approx\left|x_{1}-x_{2}^{2} \pm i x_{2}^{4}\right| \approx\left|x_{1}-x_{2}^{2}\right|+x_{2}^{4}$, hence $d\left(x, Z_{\varphi}\right) \approx$ $\min \left(\left|x_{1}\right|,\left|x_{1}-x_{2}^{2}\right|+x_{2}^{4}\right)$. For $\left|x_{1}\right| \leq 2 x_{2}^{2}$, we have $\left|x_{1}-x_{2}^{2}\right|+x_{2}^{4} \geq x_{2}^{4} \gtrsim x_{1}^{2}$ and otherwise we have $\left|x_{1}-x_{2}^{2}\right|+x_{2}^{4} \geq\left|x_{1}-x_{2}^{2}\right| \gtrsim\left|x_{1}\right|$. We derive thus the separation inequality $d\left(x, Z_{\varphi}\right) \gtrsim x_{1}^{2}=d\left(x, X_{\varphi}\right)^{2}$. On the other hand, the point $x(\epsilon)=\left(\epsilon^{2}, \epsilon\right)$ satisfies $d\left(x(\epsilon), X_{\varphi}\right)=\epsilon^{2}$ and $d\left(x(\epsilon), Z_{\varphi}\right) \leq \epsilon^{4}$, since $x(\epsilon)+\left(i \epsilon^{4}, 0\right)$ belongs to $Z_{\varphi}$. Therefore, the exponent 2 can not be lowered in the separation inequality, and we have $\tau(\varphi)=2$ whereas $d(\varphi)=4$.

(v) Note finally that if $\varphi$ is a homogeneous polynomial, we have obviously $d(\varphi)=\tau(\varphi)=1$

\section{A division theorem.}

In the sequel, $\varphi$ denotes a given germ in $\mathcal{O}\left(\mathbf{R}^{2}, 0\right)$. Using the notations of Section 1, the main result of this paper reads as follows:

Theorem 2.1. Let $M$ be a strongly regular sequence and let $g$ be a germ in $C^{\infty}\left(\mathbf{R}^{2}, 0\right)$ such that $\varphi g$ belongs to $C_{M}\left(\mathbf{R}^{2}, 0\right)$. Then $g$ belongs to $C_{M^{d(\varphi)}}\left(\mathbf{R}^{2}, 0\right)$. 
This theorem reveals that in some sense, the real arcs of $Z_{\varphi}$ do not contribute to the loss of regularity involved in the division process. We give now a few geometric lemmas to prepare the proof.

Lemma 2.2. For $1 \leq p \leq 2 p_{0}$ and $x \in\left(\mathbf{R} \times \mathbf{R}_{+}, 0\right)$, one has $d\left(x, Z_{p}\right) \gtrsim$ $|x|^{\tau_{p}^{+}}$.

Proof. Use the same argument as in [15, Lemma 3.2].

Lemma 2.3. For $2 p_{0}+1 \leq p \leq p_{1}$ and $x \in\left(\mathbf{R} \times \mathbf{R}_{+}, 0\right)$, one has $d\left(x, Z_{p}\right) \gtrsim$ $d\left(x, X_{p}\right)$.

Proof. Put $y=\left(R_{p}\left(x_{2}^{1 / m}\right), x_{2}\right)$; then $y$ belongs to $X_{p}$ and one has thus $d\left(x, X_{p}\right) \leq|x-y|=\left|x_{1}-R_{p}\left(x_{2}^{1 / m}\right)\right|$. Besides this, as in [15, Lemma 3.2], the function given by $z \mapsto\left|z_{1}-R_{p}\left(z_{2}^{1 / m}\right)\right|$ for Re $z_{2} \geq 0$ can be extended to a $C^{1}$ function $T_{p}$ in a neighborhood of 0 in $\mathbf{C}^{2}$; and $T_{p}^{-1}(0)$ is a 2-dimensional real submanifold of $\mathbf{C}^{2}$ containing $Z_{p}$. Hence we derive $d\left(x, Z_{p}\right) \geq d\left(x, T_{p}^{-1}(0)\right) \approx$ $\left|T_{p}(x)\right|=\left|x_{1}-R_{p}\left(x_{2}^{1 / m}\right)\right|$, which ends the proof.

Lemma 2.4. For $1 \leq p \leq p_{1}$ and $x \in\left(\mathbf{R} \times \mathbf{R}_{-}, 0\right)$, one has $d\left(x, Z_{p}\right) \gtrsim|x|$. Proof. Use the same argument as in [15, Lemma 3.3].

Proposition 2.5. For $x \in\left(\mathbf{R}^{2}, 0\right)$, one has $d\left(x, Z_{\varphi}\right) \gtrsim \min \left(|x|^{d(\varphi)}\right.$, $\left.d\left(x, X_{\varphi}\right)\right)$.

Proof. We remark first that we have

$$
d\left(x, Z_{\varphi}^{+}\right) \gtrsim \min \left(|x|^{d^{+}(\varphi)}, d\left(x, X_{\varphi}^{+}\right)\right) .
$$

Indeed, for $x \in\left(\mathbf{R} \times \mathbf{R}_{+}, 0\right)$, the estimate (8) is a direct consequence of Lemmas 2.2 and 2.3. For $x \in\left(\mathbf{R} \times \mathbf{R}_{-}, 0\right)$, it is a consequence of Lemma 2.4. Now, applying (8) to $\check{\varphi}(z)=\varphi\left(z_{1},-z_{2}\right)$, it is easy to obtain symmetrically

$$
d\left(x, Z_{\varphi}^{-}\right) \gtrsim \min \left(|x|^{d^{-}(\varphi)}, d\left(x, X_{\varphi}^{-}\right)\right) .
$$

The estimates (8) and (9) yield the proposition.

The previous geometric proposition, which extends [15, Proposition 3.1], is one of the two key ingredients in the proof of Theorem 2.1. The other key ingredient is of a more analytic nature; it can be stated as follows:

Proposition 2.6. Under the assumptions of Theorem 2.1, one can find constants $B_{1}$ and $B_{2}$ such that one has, for any bi-index $L$ and any point a of the real zero set $X_{\varphi}$,

$$
\left|D^{L} g(a)\right| \leq B_{1} B_{2}^{\ell} \ell ! M_{\ell}^{d(\varphi)} .
$$


The proof of Proposition 2.6 is quite technical and all of Section 3 will be devoted to it. Taking the result for granted, we shall end Section 2 by the proof of Theorem 2.1. Before this, the following important first step has to be carried out:

2.7. Reduction to the case of flat germs at the origin. Under the assumptions of Theorem 2.1, we claim that for any point $a$ in $X_{\varphi}$, there exist constants $B_{1, a}$ and $B_{2, a}$, depending on $a$, such that we have

$$
\left|D^{L} g(a)\right| \leq B_{1, a} B_{2, a}^{\ell} \ell ! M_{\ell} \text { for any } L \in \mathbf{N}^{2} .
$$

It is indeed an easy consequence of results of Chaumat-Chollet [6, Theorem 9 and Remark 17], but it can be proved by elementary arguments as well [16]. Such a statement means that division estimates hold pointwise (that is: On formal power series) without loss of regularity. However, the constants in (10) are by no means uniformly bounded with respect to $a$, whereas Proposition 2.6 gives a uniform estimate, at the cost of some loss of regularity. Nevertheless, the above estimate (10) will be useful, in the following way: Taking into account (10) for $a=0$ and Borel's theorem for Carleman classes [13], we get some $g_{0}$ in $C_{M}\left(\mathbf{R}^{2}, 0\right)$ such that $g-g_{0}$ is flat at 0 . Clearly, replacing $g$ by $g-g_{0}$ does not change the assumption nor the conclusion of Theorem 2.1. Thus we see that it is always possible to make the extra assumption that $\varphi g$ is flat at 0 , without disturbing the generality of the theorem. In the sequel, we shall use systematically this property, which, by standard arguments (see e.g., the estimate (2.17) in [15]) yields the majorization

$$
\left|D^{K}(\varphi g)(x)\right| \leq B_{3}^{k+1} k ! M_{k} h_{M}\left(B_{4}|x|\right)
$$

for any bi-index $K$ and any point $x$ in a suitable neighborhood of 0 in $\mathbf{R}^{2}$, where $B_{3}$ and $B_{4}$ are constants depending only on $g$ and $\varphi$.

2.8. Proof of Theorem 2.1. From now on, put $f=\varphi g$. Taking Proposition 2.6 into account, we still have to estimate the derivatives of $g$ at each point of $\mathbf{R}^{2} \backslash X_{\varphi}$. By the Leibniz formula, we have

$$
D^{L} g(x)=\sum_{J, K ; J+K=L} \frac{L !}{J ! K !}\left(D^{J}(1 / \varphi)(x)\right) D^{K} f(x) .
$$

The Łojasiewicz inequality asserts that there exist constants $B_{5}$ and $\nu$, with $B_{5}>0$ and $\nu \geq 1$, such that the estimate $|\varphi(z)| \geq B_{5} d\left(z, Z_{\varphi}\right)^{\nu}$ holds for any $z$ in $\left(\mathbf{C}^{2}, 0\right)$. Applying the Cauchy formula to $1 / \varphi$ on the bi-disk of radius $\frac{1}{4} d\left(x, Z_{\varphi}\right)$ centered at $x$, we derive, in virtue of Proposition 2.5 and of the previous inequality, that one has

$$
\left|D^{J}(1 / \varphi)(x)\right| \leq B_{6}^{j+1} j !\left(\min \left(|x|^{d(\varphi)}, d\left(x, X_{\varphi}\right)\right)\right)^{-(j+\nu)} .
$$

Now there are two distinct situations: 
First case: $\min \left(|x|^{d(\varphi)}, d\left(x, X_{\varphi}\right)\right)=|x|^{d(\varphi)}$. Then we proceed exactly as in [15, Theorem 2.3] to deduce from (7), (11), (12), (13) that we have $\left|D^{L} g(x)\right| \leq B_{7}^{\ell+1} \ell ! M_{\ell}^{d(\varphi)}$ for some suitable constant $B_{7}$.

Second case: $\min \left(|x|^{d(\varphi)}, d\left(x, X_{\varphi}\right)\right)=d\left(x, X_{\varphi}\right)$. Using Proposition 2.6 together with Petzsche's continuous version of the Borel theorem for Carleman classes [13], we see that for any point $a$ in $X_{\varphi}$, there exists a $C^{\infty}$ function $g_{a}$ satisfying, for any bi-index $L$,

$$
\begin{gathered}
D^{L} g_{a}(a)=D^{L} g(a) \\
\left|D^{L} g_{a}(y)\right| \leq B_{1} B_{8}\left(B_{2} B_{9}\right)^{\ell} \ell ! M_{\ell}^{d(\varphi)} \text { for any } y \text { in } \mathbf{R}^{2},
\end{gathered}
$$

with constants $B_{8}$ and $B_{9}$ depending only on $M$; in particular, uniform with respect to $a$. Put $f_{a}=\varphi g_{a}$. From (14) and (15) we derive that $f-f_{a}$ belongs to $C_{M^{d(\varphi)}}\left(\mathbf{R}^{2}, 0\right)$, is flat at $a$ and we have, in the same way as for (11),

$$
\left|D^{K}\left(f-f_{a}\right)(x)\right| \leq B_{10}^{k+1} k ! M_{k}^{d(\varphi)} h_{M^{d(\varphi)}}\left(B_{11}|x-a|\right)
$$

for any bi-index $K$, with constants $B_{10}$ and $B_{11}$ not depending on $a$. We have also $h_{M^{d(\varphi)}}\left(B_{11}|x-a|\right) \leq\left(B_{11}|x-a|\right)^{j+[\nu]+1} M_{j+[\nu]+1}^{d(\varphi)}$ and, using (4) and $(2), M_{j+[\nu]+1}^{d(\varphi)} M_{k}^{d(\varphi)} \leq B_{12}^{\ell+1} M_{\ell}^{d(\varphi)}$ for $j+k=\ell$. Pick a point $a$ in $X_{\varphi}$ satisfying $|x-a|=d\left(x, X_{\varphi}\right)$. Then, applying (12) with $g-g_{a}$ instead of $g$ and $f-f_{a}$ instead of $f$, one gets, in virtue of (7), (13), (16) and the previous inequalities, $\left|D^{L}\left(g-g_{a}\right)(x)\right| \leq B_{13}^{\ell+1} \ell ! M_{\ell}^{d(\varphi)}$. Taking (15) into account, we derive $\left|D^{L} g(x)\right| \leq B_{14}^{\ell+1} \ell ! M_{\ell}^{d(\varphi)}$ for some suitable $B_{14}$, thus ending the proof.

We turn now to the proof of Proposition 2.6.

\section{Estimates along the zero set.}

We shall use the analytic vector field $\mathbf{Y}=m^{-1} x_{2}^{1-m} \partial_{2}$ on $\mathbf{R} \times \mathbf{R}^{*}$, in such a way that we have, for any $h$ in $C^{\infty}\left(\mathbf{R}^{2}, 0\right)$ and any $x$ in $\left(\mathbf{R} \times \mathbf{R}^{*}, 0\right)$,

$$
\left(\partial_{2} h\right)\left(x_{1}, x_{2}^{m}\right)=\mathbf{Y}\left(h\left(x_{1}, x_{2}^{m}\right)\right) .
$$

The following proposition will play a crucial role.

Proposition 3.1. Let $R$ be an element of $\mathcal{O}(\mathbf{R}, 0)$ satisfying $\omega(R) \geq m$ and let $N$ be an integer with $N \geq 1$. Consider a germ $g$ in $C^{\infty}\left(\mathbf{R}^{2}, 0\right)$ and assume that for every real $\beta>0$, there exist constants $C_{1}$ and $C_{2}$, depending on $\beta$, such that for any point $x$ in $\left(\mathbf{R}^{2}, 0\right)$ satisfying $\left|x_{1}\right|<\beta\left|x_{2}\right|^{m}$, the estimate

$$
\left|\partial_{1}^{k} \mathbf{Y}^{\ell}\left(\left(x_{1}-R\left(x_{2}\right)\right)^{N} G(x)\right)\right| \leq C_{1}^{k+\ell+1}(k+\ell) ! M_{k+\ell} h_{M}\left(C_{2}\left|x_{2}\right|^{m}\right)
$$

holds for every bi-index $(k, \ell)$ in $\mathbf{N}^{2}$. Then, for every real $\beta>0$, there exist constants $C_{3}$ and $C_{4}$, depending only on $C_{1}, C_{2}, R, N, m, \beta$ and $M$, such 
that for any point $x$ in $\left(\mathbf{R}^{2}, 0\right)$ satisfying $\left|x_{1}\right|<\beta\left|x_{2}\right|^{m}$, the estimate

$$
\left|\partial_{1}^{k} \mathbf{Y}^{\ell} G(x)\right| \leq C_{3}^{k+\ell+1}(k+\ell) ! M_{k+\ell} h_{M}\left(C_{4}\left|x_{2}\right|^{m}\right)
$$

holds for every bi-index $(k, \ell)$ in $\mathbf{N}^{2}$.

Proof. The proof is quite technical and it will be cut into several lemmas. We define, for $0 \leq t \leq 1$ and $x_{2} \in \mathbf{R}^{*}$,

$$
Q\left(x_{2}, t\right)=(1-t) m^{-1} x_{2}^{1-m} R^{\prime}\left(x_{2}\right) .
$$

We also put, for $0 \leq t \leq 1$ and $x \in\left(\mathbf{R}^{2}, 0\right)$,

$$
y=y(x, t)=\left(t x_{1}+(1-t) R\left(x_{2}\right), x_{2}\right) .
$$

When $x$ satisfies $\left|x_{1}\right|<\beta\left|x_{2}\right|^{m}$, one gets, for some constant $C_{5}$ depending only on $R$,

$$
\left|y_{1}\right|<\beta\left|x_{2}\right|^{m}+\left|R\left(x_{2}\right)\right|<\left(\beta+C_{5}\right)\left|y_{2}\right|^{m}
$$

since $y_{2}=x_{2}$ and $\omega(R) \geq m$.

Lemma 3.2. For any $x_{2}$ in $\left(\mathbf{R}^{*}, 0\right)$, any $p$ in $\mathbf{N}$ and any $t$ in $[0,1]$, one has

$$
\left|\mathbf{Y}^{p} Q\left(x_{2}, t\right)\right| \leq C_{6} C_{7}^{p} p !\left|x_{2}\right|^{-m p},
$$

where the constants $C_{6}$ and $C_{7}$ depend only on $R$ and $m$.

Proof. The assumption $\omega(R) \geq m$ ensures that $Q$ is analytic for $x_{2}$ in a neighborhood of 0 . We have therefore

$$
\left|\partial_{2}^{j} Q\left(x_{2}, t\right)\right| \leq C_{8} C_{9}^{j} j !
$$

for any $j \in \mathbf{N}$, where the constants $C_{8}$ and $C_{9}$ depend only on $R$ and $m$. Besides this, an induction on the integer $p$ gives the expansion

$$
\mathbf{Y}^{p}=\sum_{j=1}^{p} \mathbf{Y}_{j}^{(p)}\left(x_{2}\right) \partial_{2}^{j}
$$

where the coefficients $\mathbf{Y}_{j}^{(p)}\left(x_{2}\right)$ satisfy the estimate

$$
\left|\mathbf{Y}_{j}^{(p)}\left(x_{2}\right)\right| \leq C_{10}^{p+1}(p-j) !\left|x_{2}\right|^{j-m p} \text { for } j=1, \ldots, p .
$$

This estimate can be obtained as a very special case of [14, Proposition 2.5]. Finally, Lemma 3.2 is easy to deduce from (18), (19), (20).

Lemma 3.3. For any germ $h$ in $C^{\infty}\left(\mathbf{R}^{2}, 0\right)$, any point $x$ in $\left(\mathbf{R} \times \mathbf{R}^{*}, 0\right)$, any integer $\ell \geq 1$ and any real $t$ in $[0,1]$, one has

$$
\mathbf{Y}^{\ell}(h(y(x, t)))=\sum_{(i, j) \in \mathbf{N}^{2} ; 1 \leq i+j \leq \ell} \Gamma_{i, j}^{(\ell)}\left(x_{2}, t\right)\left(\partial_{1}^{i} \mathbf{Y}^{j} h\right)(y(x, t)),
$$


where the coefficients $\Gamma_{i, j}^{(\ell)}\left(x_{2}, t\right)$ satisfy, for every integer $k \geq 0$, the estimate

$$
\left|\mathbf{Y}^{k} \Gamma_{i, j}^{(\ell)}\left(x_{2}, t\right)\right| \leq\left(4 C_{11}\right)^{\ell}\left(2 C_{7}\right)^{k+\ell-i-j}(k+\ell-i-j) !\left|x_{2}\right|^{-m(k+\ell-i-j)}
$$

with $C_{11}=\max \left(1, C_{6}\right)$.

Proof. We proceed by induction on $\ell$. A simple computation shows that

$$
\mathbf{Y}(h(y(x, t)))=Q\left(x_{2}, t\right)\left(\partial_{1} h\right)(y(x, t))+(\mathbf{Y} h)(y(x, t)),
$$

which proves $(21)$ for $\ell=1$, with $\Gamma_{1,0}^{(1)}\left(x_{2}, t\right)=Q\left(x_{2}, t\right)$ and $\Gamma_{0,1}^{(1)}\left(x_{2}, t\right)=1$. In this case, the estimate (22) is a direct consequence of Lemma 3.2. Now, an integer $\ell$ being given, the computation of $\mathbf{Y}\left(\mathbf{Y}^{\ell}(h(y(x, t)))\right)$ starting from the expansion (21) gives

$$
\Gamma_{i, j}^{(\ell+1)}\left(x_{2}, t\right)=\mathbf{Y} \Gamma_{i, j}^{(\ell)}\left(x_{2}, t\right)+\Gamma_{i, j-1}^{(\ell)}\left(x_{2}, t\right)+Q\left(x_{2}, t\right) \Gamma_{i-1, j}^{(\ell)}\left(x_{2}, t\right),
$$

where we have put $\Gamma_{i, j}^{(\ell)}=0$ for $i<0$ or $j<0$, for $i=j=0$, and for $i+j>\ell$. In virtue of the Leibniz formula, (23) yields

$$
\begin{aligned}
\mathbf{Y}^{k} \Gamma_{i, j}^{(\ell+1)}\left(x_{2}, t\right)= & \mathbf{Y}^{k+1} \Gamma_{i, j}^{(\ell)}\left(x_{2}, t\right)+\mathbf{Y}^{k} \Gamma_{i, j-1}^{(\ell)}\left(x_{2}, t\right) \\
& +\sum_{p+q=k} \frac{k !}{p ! q !}\left(\mathbf{Y}^{p} Q\left(x_{2}, t\right)\right)\left(\mathbf{Y}^{q} \Gamma_{i-1, j}^{(\ell)}\left(x_{2}, t\right)\right) .
\end{aligned}
$$

By induction assumption, both terms $\mathbf{Y}^{k+1} \Gamma_{i, j}^{(\ell)}\left(x_{2}, t\right)$ and $\mathbf{Y}^{k} \Gamma_{i, j-1}^{(\ell)}\left(x_{2}, t\right)$ are majorized by $\left(4 C_{11}\right)^{\ell}\left(2 C_{7}\right)^{k+\ell+1-i-j}(k+\ell+1-i-j) !\left|x_{2}\right|^{-m(k+\ell+1-i-j)}$. For each term occuring with $p+q=k$ in the sum at the right-hand side of (24), we have also, in virtue of Lemma 3.2,

$$
\begin{aligned}
\left|\left(\mathbf{Y}^{p} Q\left(x_{2}, t\right)\right)\left(\mathbf{Y}^{q} \Gamma_{i-1, j}^{(\ell)}\left(x_{2}, t\right)\right)\right| \leq C_{6} C_{7}^{p} p !\left|x_{2}\right|^{-m p}\left(4 C_{11}\right)^{\ell}\left(2 C_{7}\right)^{q+\ell+1-i-j} \\
\cdot(q+\ell+1-i-j) !\left|x_{2}\right|^{-m(q+\ell+1-i-j)} .
\end{aligned}
$$

Using the elementary fact $p !(q+\ell+1-i-j) ! \leq(k+\ell+1-i-j)$ !, we deduce, from these estimates and from the definition of $C_{11}$, that we have

$$
\begin{aligned}
& \left|\mathbf{Y}^{k} \Gamma_{i, j}^{(\ell+1)}\left(x_{2}, t\right)\right| \\
& \quad \leq\left(4 C_{11}\right)^{\ell}\left(2 C_{7}\right)^{k+\ell+1-i-j}(k+\ell+1-i-j) !\left|x_{2}\right|^{-m(k+\ell+1-i-j)} \mathcal{S},
\end{aligned}
$$

with $\mathcal{S}=2+C_{11} \sum_{p+q=k} \frac{k !(q+\ell+1-i-j) !}{q !(k+\ell+1-i-j) !} 2^{-p}$. It is easy to check that one has

$$
\frac{k !(q+\ell+1-i-j) !}{q !(k+\ell+1-i-j) !} \leq 1 \text { for } 0 \leq q \leq k \text { and } i+j \leq \ell+1 .
$$

We have thus $\mathcal{S} \leq 2+C_{11} \sum_{0 \leq p \leq k} 2^{-p} \leq 2+2 C_{11} \leq 4 C_{11}$. Therefore the estimate (25) completes the induction and Lemma 3.3 is proved. 


\subsection{End of the proof of Proposition 3.1. Put}

$$
F(x)=\left(x_{1}-R\left(x_{2}\right)\right)^{N} G(x)
$$

and consider the real analytic change of coordinates near the origin of $\mathbf{R}^{2}$ given by $u_{1}=x_{1}-R\left(x_{2}\right), u_{2}=x_{2}$. In this system of coordinates, (26) becomes

$$
F \circ \psi(u)=u_{1}^{N}(G \circ \psi)(u) \text { with } \psi(u)=\left(u_{1}+R\left(u_{2}\right), u_{2}\right) .
$$

This yields easily, for $x=\psi(u)$,

$$
\begin{aligned}
G \circ \psi(u) & =\int_{0}^{1} \frac{(1-t)^{N-1}}{(N-1) !} \frac{\partial^{N}(F \circ \psi)}{\partial u_{1}^{N}}\left(t u_{1}, u_{2}\right) d t \\
& =\int_{0}^{1} \frac{(1-t)^{N-1}}{(N-1) !}\left(\partial_{1}^{N} F\right)(y(x, t)) d t .
\end{aligned}
$$

Taking into account Lemma 3.3 and the fact that $\partial_{1}$ and $\mathbf{Y}$ commute, we get thus

$$
\partial_{1}^{k} \mathbf{Y}^{\ell} G(x)=\sum_{1 \leq i+j \leq \ell} \int_{0}^{1} \frac{t^{k}(1-t)^{N-1}}{(N-1) !} \Gamma_{i, j}^{(\ell)}\left(x_{2}, t\right)\left(\partial_{1}^{i+k+N} \mathbf{Y}^{j} F\right)(y(x, t)) d t .
$$

Now, the assumptions on $F$ in Proposition 3.1, together with the estimate (17) on $y(x, t)$, ensure that one has

$$
\begin{gathered}
\left|\left(\partial_{1}^{i+k+N} \mathbf{Y}^{j} F\right)(y(x, t))\right| \leq C_{12}^{i+j+k+N+1}(i+j+k+N+1) ! \\
\cdot M_{i+j+k+N+1} h_{M}\left(C_{13}\left|x_{2}\right|^{m}\right)
\end{gathered}
$$

for any $t$ in $[0,1]$ and any $x$ in $\left(\mathbf{R}^{2}, 0\right)$ satisfying $\left|x_{1}\right|<\beta\left|x_{2}\right|^{m}$, where $C_{12}$ and $C_{13}$ are suitable constants depending on $\beta$. Besides this, the estimate (22) in Lemma 3.3 gives

$$
\left|\Gamma_{i, j}^{(\ell)}\left(x_{2}, t\right)\right| \leq C_{14}^{\ell+1}(\ell-i-j) !\left|x_{2}\right|^{-m(\ell-i-j)}
$$

with $C_{14}=\max \left(4 C_{11}, 2 C_{7}\right)$. We have also, in virtue of (6), the inequality

$$
h_{M}\left(C_{13}\left|x_{2}\right|^{m}\right) \leq\left(C_{15}\left|x_{2}\right|^{m}\right)^{\ell-i-j} M_{\ell-i-j} h_{M}\left(C_{15}\left|x_{2}\right|^{m}\right)
$$

with $C_{15}=\rho(2) C_{13}$. Finally, we have the elementary estimates $(i+j+k+$ $N+1) !(\ell-i-j) ! \leq(k+\ell+N+1) ! \leq 2^{k+\ell+N+1}(k+\ell) !(N+1) !$ as well as $M_{i+j+k+N+1} M_{\ell-i-j} \leq M_{k+\ell+N+1} \leq \bar{A}^{k+\ell+N+1} M_{k+\ell} M_{N+1}$ by (2) and (4). Thus, gathering (28), (29) and (30) in (27), we obtain the desired estimate of Proposition 3.1.

Now, going back to Section 2, we have to obtain bounds for the derivatives of $g$ on the real zero set $X_{\varphi}$. In this purpose, we shall carry out the estimates on $X_{p}$ for any fixed $p$ with $2 p_{0}+1 \leq p \leq p_{1}$. This will yield a global estimate, first on $X_{\varphi}^{+}$, then on the whole of $X_{\varphi}$ by symmetry. Of course, if 
$2 p_{0}=p_{1}$ (which means $X_{\varphi}^{+}=\{0\}$ ), we have nothing to do. Otherwise, it is clearly enough (since $\left.X_{p} \cap(\mathbf{R} \times\{0\})=\{0\}\right)$ to prove a uniform bound on $X_{p} \cap\left(\mathbf{R} \times \mathbf{R}_{+}^{*}\right)$. In the sequel, $a$ denotes a point on this set; in other words, we have

$$
a_{2}>0 \quad \text { and } \quad a_{1}=R_{p}\left(a_{2}^{1 / m}\right) .
$$

Let $H$ be the half-plane $\{w \in \mathbf{C} ; \operatorname{Re} w>0\}$. For any $z$ in $(\mathbf{C} \times H, 0)$, put

$$
\widetilde{\varphi}(z)=u(z) \prod_{q=1}^{2 p_{0}}\left(z_{1}-U_{q}\left(z_{2}^{1 / m}\right)\right)^{n_{q}} .
$$

We also define, for any $x$ in $\left(\mathbf{R} \times \mathbf{R}_{+}^{*}, 0\right)$,

$$
\widetilde{g}(x)=\widetilde{\varphi}(x) g(x),
$$

in such a way that we have

$$
\widetilde{g}(x) \prod_{q=2 p_{0}+1}^{p_{1}}\left(x_{1}-R_{q}\left(x_{2}^{1 / m}\right)\right)^{n_{q}}=(\varphi g)(x) .
$$

Lemma 3.5. There exist constants $C_{16}$ and $C_{17}$ such that one has, for any bi-index $L$,

$$
\left|D^{L} \widetilde{g}(a)\right| \leq C_{16}^{\ell+1} \ell ! M_{\ell} h_{M}\left(C_{17}|a|\right) .
$$

Proof. For $j=2 p_{0}+1, \ldots, p_{1}+1$, put

$$
G_{j}(x)=\widetilde{g}\left(x_{1}, x_{2}^{m}\right) \prod_{j \leq q \leq p_{1}}\left(x_{1}-R_{q}\left(x_{2}\right)\right)^{n_{q}} .
$$

Using (32) and (33), the functions $G_{j}$ defined above for $x \in\left(\mathbf{R} \times \mathbf{R}_{+}^{*}, 0\right)$ are easily seen to be, in fact, of class $C^{\infty}$ in a whole neighborhood of 0 in $\mathbf{R}^{2}$. Moreover, one has

$$
\begin{gathered}
\left(x_{1}-R_{j}\left(x_{2}\right)\right)^{n_{j}} G_{j+1}(x)=G_{j}(x), \\
G_{2 p_{0}+1}(x)=(\varphi g)\left(x_{1}, x_{2}^{m}\right) .
\end{gathered}
$$

In particular, we have $\partial_{1}^{k} \mathbf{Y}^{\ell} G_{2 p_{0}+1}(x)=\left(\partial_{1}^{k} \partial_{2}^{\ell}(\varphi g)\right)\left(x_{1}, x_{2}^{m}\right)$. In virtue of the estimate (11) and the induction relations (34) and (35), it is possible to apply Proposition 3.1 repeatedly with $j=2 p_{0}+1, \ldots, p_{1}$. We get thus

$$
\left|\partial_{1}^{k} \mathbf{Y}^{\ell} G_{p_{1}+1}(x)\right| \leq C_{18}^{k+\ell+1}(k+\ell) ! M_{k+\ell} h_{M}\left(C_{19}\left|x_{2}\right|^{m}\right)
$$

for any $(k, \ell)$ in $\mathbf{N}^{2}$ and any $x$ in $\left(\mathbf{R}^{2}, 0\right)$ satisfying

$$
\left|x_{1}\right|<\beta\left|x_{2}\right|^{m}
$$

with suitable constants $C_{18}$ and $C_{19}$ depending on $\beta$. Finally, the point $x=\left(a_{1}, a_{2}^{1 / m}\right)$ satisfies (37) for some $\beta>0$ depending only on $\varphi$, in virtue of (31) and since $\omega\left(R_{p}\right) \geq m$. Therefore, applying (36) for this choice of $x$ completes the proof of the lemma, since we have $G_{p_{1}+1}(x)=\widetilde{g}\left(x_{1}, x_{2}^{m}\right)$. 
In order to estimate the derivatives of $g=\widetilde{g} / \widetilde{\varphi}$ at the point $a$, we finally need some bounds on $1 / \widetilde{\varphi}$. Such bounds are obtained in the following lemma. Put first

$$
\widetilde{Z}=\bigcup_{1 \leq q \leq 2 p_{0}} Z_{q}
$$

Lemma 3.6. One has

$$
\begin{gathered}
|\widetilde{\varphi}(z)| \geq C_{20} d(z, \widetilde{Z})^{\widetilde{\nu}} \text { for any } z \in(\mathbf{C} \times H, 0), \\
d(a, \widetilde{Z}) \geq C_{21}|a|^{d^{+}(\varphi)},
\end{gathered}
$$

with $\widetilde{\nu}=\sum_{1 \leq q \leq 2 p_{0}} n_{q}$ and constants $C_{20}, C_{21}$ depending only on $\varphi$.

Proof. For $z \in \mathbf{C} \times H$, the point $z(q)=\left(U_{q}\left(z_{2}^{1 / m}\right), z_{2}\right)$ belongs to $Z_{q}$, hence $d\left(z, Z_{q}\right) \leq|z-z(q)|=\left|z_{1}-U_{q}\left(z_{2}^{1 / m}\right)\right|$. One has also $d(z, \widetilde{Z}) \leq d\left(z, Z_{q}\right)$ for $q=1, \ldots, 2 p_{0}$. Taking $\widetilde{\nu}$ as indicated, we get thus

$$
d(z, \widetilde{Z})^{\widetilde{\nu}} \leq \prod_{1 \leq q \leq 2 p_{0}} d\left(z, Z_{q}\right)^{n_{q}} \leq \prod_{1 \leq q \leq 2 p_{0}}\left|z_{1}-U_{q}\left(z_{2}^{1 / m}\right)\right|^{n_{q}},
$$

which, by definition of $\widetilde{\varphi}$, proves (38). We have also, using (31) and the same sort of arguments as in Lemma 2.3, $d\left(a, Z_{q}\right) \gtrsim\left|a_{1}-U_{q}\left(a_{2}^{1 / m}\right)\right|=$ $\left|R_{p}\left(a_{2}^{1 / m}\right)-U_{q}\left(a_{2}^{1 / m}\right)\right| \geq\left|S_{q}\left(a_{2}^{1 / m}\right)\right| \gtrsim a_{2}^{\mu_{q} / m}$ for $q=1, \ldots, 2 p_{0}$. The estimate (39) follows, since (31) implies $|a| \lesssim a_{2}$.

Lemma 3.7. For any bi-index $J$, one has the estimate

$$
\left|D^{J}(1 / \widetilde{\varphi})(a)\right| \leq C_{22}^{j+1} j !|a|^{-d^{+}(\varphi)(j+\widetilde{\nu})},
$$

where the constant $C_{22}$ depends only on $\varphi$.

Proof. Since we have $|a| \lesssim a_{2}$, we derive $d(a, \widetilde{Z}) \lesssim a_{2}$. Therefore, for a sufficiently small $\epsilon>0$, the bi-disk of radius $\epsilon d(a, \widetilde{Z})$ centered at $a$ lies in $\mathbf{C} \times H$ and we can apply the Cauchy formula, together with the estimate (38) of Lemma 3.6, to obtain

$$
\left|D^{J}(1 / \widetilde{\varphi})(a)\right| \leq C_{23}^{j+1} j ! d(a, \widetilde{Z})^{-(j+\widetilde{\nu})}
$$

for some suitable $C_{23}$. Then the estimate (39) of Lemma 3.6 yields the conclusion.

3.8. End of the proof of Proposition 2.6. It is now enough to estimate $D^{L}(\widetilde{g} / \widetilde{\varphi})(a)$ by means of the Leibniz formula, with the help of Lemmas 3.5 and 3.7. By arguments similar to those in Subsection 2.8, we obtain

$$
\left|D^{L} g(a)\right| \leq C_{24}^{\ell+1} \ell ! M_{\ell}^{d^{+}(\varphi)}
$$


for any $L$ in $\mathbf{N}^{2}$ and any $a$ in $X_{p}$, with $2 p_{0}+1 \leq p \leq p_{1}$, hence any $a$ belonging to $X_{\varphi}^{+}$. We have symmetrically

$$
\left|D^{L} g(a)\right| \leq C_{25}^{\ell+1} \ell ! M_{\ell}^{d^{-}(\varphi)}
$$

when $a$ belongs to $X_{\varphi}^{-}$. Clearly, Proposition 2.6 follows.

\section{Further results and comments.}

We shall discuss here first the sharpness of Theorem 2.1. In the particular situation $Z_{\varphi} \cap \mathbf{R}^{2}=\{0\}$, it can be obtained as a consequence of [15, Theorem 2.6]. The result is in fact optimal in full generality, as shown by the following theorem.

Theorem 4.1. For any germ $\varphi$ in $\mathcal{O}\left(\mathbf{R}^{2}, 0\right)$ and any strongly regular sequence $M$, one can always find a germ $g$ in $C_{M^{d(\varphi)}}\left(\mathbf{R}^{2}, 0\right)$, flat at the origin, such that $\varphi g$ belongs to $C_{M}\left(\mathbf{R}^{2}, 0\right)$ and $g$ does not belong to any Carleman class strictly smaller than $C_{M^{d(\varphi)}}\left(\mathbf{R}^{2}, 0\right)$.

Proof. It goes partly along the same lines as [15, Subsection 3.3], so we shall skip some details. Without losing generality, we can assume $d(\varphi)=d^{+}(\varphi)$. Put

$$
g(x)=\frac{\eta\left(x_{2}\right)}{\widetilde{\varphi}\left(x_{1}, x_{2}\right)} \text { for } x_{2}>0 \text { and } g(x)=0 \text { for } x_{2} \leq 0,
$$

where $\eta$ is the special flat germ in $C_{M}(\mathbf{R}, 0)$ given by [15, Lemma 3.6]; and $\widetilde{\varphi}$ has been defined by (32). Proceeding as in [15], it is readily seen that $g$ belongs to $C_{M^{d(\varphi)}}\left(\mathbf{R}^{2}, 0\right)$ but not to any smaller class. Now, the only thing which remains to be checked is that $\varphi g$ belongs to $C_{M}\left(\mathbf{R}^{2}, 0\right)$. By definition of $g$ in (40), it is enough to obtain estimates in $\mathbf{R} \times \mathbf{R}_{+}$. For $x_{2} \geq 0$, we have

$$
(\varphi g)\left(x_{1}, x_{2}^{m}\right)=\eta\left(x_{2}^{m}\right) \prod_{q=2 p_{0}+1}^{p_{1}}\left(x_{1}-R_{q}\left(x_{2}\right)\right)^{n_{q}} .
$$

Now, using the flatness of $\eta$ and the definition of $\mathbf{Y}$ at the beginning of Section 3, we have

$$
\left|\mathbf{Y}^{\ell}\left(\eta\left(x_{2}^{m}\right)\right)\right| \leq D_{1}^{\ell+1} \ell ! M_{\ell} h_{M}\left(D_{2}\left|x_{2}\right|^{m}\right)
$$

for any positive integer $\ell$, where $D_{1}$ and $D_{2}$ are suitable constants. Besides this, in the same way as for Lemma 3.2, we have

$$
\left|\mathbf{Y}^{\ell} R_{q}\left(x_{2}\right)\right| \leq D_{3}^{\ell+1} \ell !\left|x_{2}\right|^{-m \ell}
$$

for $q=2 p_{0}+1, \ldots, p_{1}$. Using (41), (42), (43) and the Leibniz formula, we obtain, by the same sort of arguments as in the end of the proof of Proposition 3.1,

$$
\left|\partial_{1}^{k} \mathbf{Y}^{\ell}\left((\varphi g)\left(x_{1}, x_{2}^{m}\right)\right)\right| \leq D_{4}^{k+\ell+1}(k+\ell) ! M_{k+\ell} h_{M}\left(D_{5}\left|x_{2}\right|^{m}\right)
$$


for any bi-index $(k, \ell)$. Applying (44) with $x=\left(y_{1}, y_{2}^{1 / m}\right)$, where $y$ is any given point of $\mathbf{R} \times \mathbf{R}_{+}$, we get thus

$$
\left|\partial_{1}^{k} \partial_{2}^{\ell}(\varphi g)(y)\right| \leq D_{4}^{k+\ell+1}(k+\ell) ! M_{k+\ell} h_{M}\left(D_{5}\left|y_{2}\right|\right),
$$

hence the desired result.

Next we describe an application of Theorem 2.1 to the study of closed ideals in ultradifferentiable classes. It requires only a few basic facts about topology on Carleman classes, recalled hereafter. For any smoothly bounded neighborhood $U$ of 0 in $\mathbf{R}^{n}$ and any real $\sigma>0$, let $C_{M, \sigma}(\bar{U})$ be the set of those functions $f$ belonging to $C^{\infty}(\bar{U})$ and such that there exists a constant $C_{f}>0$ for which we have $\left|D^{L} f(x)\right| \leq C_{f} \sigma^{\ell} \ell ! M_{\ell}$ for any $x$ in $U$ and any multi-index $L$. This set is a Banach space for the norm $\|f\|_{U, \sigma}$ defined as the smallest possible $C_{f}$ in the previous condition. One can then define the Carleman class $C_{M}(\bar{U})$ as the inductive limit of all spaces $C_{M, \sigma}(\bar{U})$ for increasing $\sigma>0$ (we refer the reader to [9] for a detailed study of such spaces). We shall say that the ideal $I_{\varphi, M}$ generated by a real-analytic germ $\varphi$ in $C_{M}\left(\mathbf{R}^{n}, 0\right)$ is closed if any germ which, for some suitable neighborhood $U$ of 0 , belongs to the closure of the ideal $\varphi C_{M}(\bar{U})$ generated by $\varphi$ in $C_{M}(\bar{U})$, actually belongs to $I_{\varphi, M}$ (that is, it belongs to $\varphi C_{M}(\bar{V})$ for some neighborhood $V$ of 0 , maybe smaller than $U$ ). Now, the following result extends [15, Corollary 4.2] to the case of general zero sets. Note that we state it for germs in order to stay in the setting of the paper, but a corresponding theorem for functions can be written as well.

Theorem 4.2. The ideal $I_{\varphi, M}$ generated by $\varphi$ in $C_{M}\left(\mathbf{R}^{2}, 0\right)$ is closed if and only if one has $d(\varphi)=1$.

Proof. First, any element $f$ of $C_{M}\left(\mathbf{R}^{2}, 0\right)$ belonging belongs to the closure of $\varphi C_{M}(\bar{U})$ in $C_{M}(\bar{U})$ for some neighborhood $U$ of 0 , also belongs to the $C^{\infty}$-closure of $\varphi C^{\infty}(U)$. The Łojasiewicz-Malgrange division theorem [10], [11] ensures that the latter ideal is $C^{\infty}$-closed, thus $f$ can be written $\varphi g$ for some $g$ in $C^{\infty}\left(\mathbf{R}^{2}, 0\right)$. Assuming $d(\varphi)=1$, Theorem 2.1 implies that $g$ belongs to $C_{M}\left(\mathbf{R}^{2}, 0\right)$. Therefore the condition is sufficient for closedness. Now, we prove the converse: Let $g$ be the germ constructed in Theorem 4.1. From the argument of Subsection 2.7, we deduce that for any point $a$ in a suitable neighborhood $U$ of 0 , there exists some function $f_{a}$ belonging to the ideal $\varphi C_{M}(\bar{U})$ and such that $\varphi g-f_{a}$ is flat at the point $a$. Applying the ultradifferentiable version of Whitney's spectral theorem [6] and assuming that $I_{\varphi, M}$ is closed, we derive that $\varphi g$ belongs to $I_{\varphi, M}$, hence $g$ belongs to $C_{M}\left(\mathbf{R}^{2}, 0\right)$. In virtue of Theorem 4.1, this implies $d(\varphi)=1$.

4.3. A discussion of the higher-dimensional case. We shall see here that Theorem 4.1 can be extended to germs in $\mathbf{R}^{n}$ with $n \geq 3$, provided that, 
as in [15], we assume to be in the special situation $Z_{\varphi} \cap \mathbf{R}^{n}=\{0\}$, and we replace consequently $d(\varphi)$ by the Lojasiewicz exponent $\tau(\varphi)$ for the regular separation between $Z_{\varphi}$ and $\mathbf{R}^{n}$. As a consequence, the answer to Problem 2.7 in $[\mathbf{1 5}]$ is affirmative and the assumption $n=2$ is no longer necessary in [15, Theorem 2.6]. Note that Theorem 4.2 extends as well, since it reduces to $[\mathbf{1 5}$, Corollary 4.2$]$ in this situation. The scheme of proof, pointed out by Bierstone [1], is as follows:

- First, using the curve-selecting lemma as in [2, Theorem 6.4] or [3], one can find a real-analytic curve $\sigma:[-\varepsilon, \varepsilon] \rightarrow Z_{\varphi}$, with $\sigma(0)=0$, which realizes the Łojasiewicz exponent $\tau(\varphi)$; in other words, such that $\tau(\varphi)$ is also the Eojasiewicz exponent for the regular separation between $\mathbf{R}^{n}$ and $\sigma([-\varepsilon, \varepsilon])$. Now, consider the germ of complex curve $\Sigma$ defined as the image of $\sigma:(\mathbf{C}, 0) \rightarrow\left(\mathbf{C}^{n}, 0\right)$ and denote by $\tau^{+}(\varphi)$ the Eojasiewicz exponent for the regular separation between $\mathbf{R}^{n}$ and $Z_{\varphi}^{+}=Z_{\varphi} \cap\{z \in$ $\left.\mathbf{C}^{n} ; \operatorname{Re} z_{n} \geq 0\right\}$. We have clearly $\sigma([-\varepsilon, \varepsilon]) \subset \Sigma \subset Z_{\varphi}$, thus $\tau^{+}(\varphi)$ is also the Eojasiewicz exponent for the regular separation between $\mathbf{R}^{n}$ and $\Sigma^{+}=\Sigma \cap\left\{z \in \mathbf{C}^{n} ; \operatorname{Re} z_{n} \geq 0\right\}$. Write $\sigma(t)=\left(\sigma_{1}(t), \ldots, \sigma_{n}(t)\right)$ for $t \in(\mathbf{C}, 0)$. Up to analytic changes of coordinates, we can assume that $\sigma_{n}(t)=t^{m}$ for some integer $m \geq 1$, and that $\omega\left(\sigma_{j}\right) \geq m$ for $j=1, \ldots, n-1$. Put $\sigma_{j}(t)=X_{j}(t)+i Y_{j}(t)$, where both $X_{j}, Y_{j}$ belong to $\mathcal{O}(\mathbf{R}, 0)$; and put $\mu=\inf \left\{\omega\left(Y_{j}\right) ; j=1, \ldots, n-1\right\}$ (the integer $\mu$ is finite, since we have $\left.\Sigma \cap \mathbf{R}^{n}=\{0\}\right)$. We claim that

$$
\tau^{+}(\varphi)=\mu / m \text {. }
$$

Indeed, this can be proved in the same way as [15, Lemma 3.2], reasoning with $\Sigma^{+}$instead of $Z_{\varphi}^{+}$: Consider the map $z \mapsto T(z)=$ $\left(T_{1}(z), \ldots, T_{n-1}(z)\right)$, where each $T_{j}$ is a $C^{1}$ extension to $\left(\mathbf{C}^{n}, 0\right)$ of the function $z \mapsto z_{j}-\sigma_{j}\left(z_{n}^{1 / m}\right)$, first defined on $\left\{z \in \mathbf{C}^{n} ; \operatorname{Re} z_{n} \geq 0\right\}$. One has then, for $x \in\left(\mathbf{R}^{n}, 0\right)$, the estimate $d\left(x, T^{-1}(0)\right) \approx\left|T_{1}(x)\right|+\cdots+$ $\left|T_{n-1}(x)\right|$ with $\left|T_{j}(x)\right| \approx\left|x_{j}-X_{j}\left(x_{n}^{1 / m}\right)\right|+\left|Y_{j}\left(x_{n}^{1 / m}\right)\right|$, and arguments similar to those in [15] can be applied.

- Now, by the definition of $\mu$, we can write $Y_{j}\left(z_{n}\right)=z_{n}^{\mu} Y_{j}^{*}\left(z_{n}\right)$ for $j=$ $1, \ldots, n-1$, where each $Y_{j}^{*}$ belongs to $\mathcal{O}(\mathbf{R}, 0)$. Put $X=\left(X_{1}, \ldots\right.$, $\left.X_{n-1}\right), Y^{*}=\left(Y_{1}^{*}, \ldots, Y_{n-1}^{*}\right)$ and, for $\left(t, z_{n}\right) \in\left(\mathbf{C}^{2}, 0\right)$,

$$
\varphi^{*}\left(t, z_{n}\right)=\varphi\left(X\left(z_{n}\right)+t Y^{*}\left(z_{n}\right), z_{n}\right) .
$$

Note that $\varphi^{*}\left(i z_{n}^{\mu}, z_{n}^{m}\right)=\varphi\left(\sigma\left(z_{n}\right)\right)=0$ for any $z_{n} \in(\mathbf{C}, 0)$. Moreover, for any real $x_{n}$, the roots of the real-analytic germ $t \mapsto \varphi^{*}\left(t, x_{n}^{m}\right)$ are non-real, pairwise conjugate. Therefore, this germ is divisible by $\left(t-i x_{n}^{\mu}\right)\left(t+i x_{n}^{\mu}\right)=t^{2}+x_{n}^{2 \mu}$. Now, as in the proof of Theorem 4.1, we can assume $\tau(\varphi)=\tau^{+}(\varphi)$ and consider the germ $g$ defined, for 
$x \in\left(\mathbf{R}^{n}, 0\right)$, by

$$
g(x)=\eta\left(x_{n}\right) / \varphi(x) \text { for } x_{n}>0 \text { and } g(x)=0 \text { for } x_{n} \leq 0 .
$$

In virtue of [15, Theorem 2.6], $g$ belongs to $C_{M^{\tau(\varphi)}}\left(\mathbf{R}^{n}, 0\right)$ and it is flat at the origin. Conversely, assuming that $g$ belongs to $C_{M^{s}}\left(\mathbf{R}^{n}, 0\right)$ and replacing $x$ by $\left(X\left(x_{n}\right)+t Y^{*}\left(x_{n}\right), x_{n}^{m}\right)$ in (46), we get

$$
\eta\left(x_{n}^{m}\right)=\left(t^{2}+x_{n}^{2 \mu}\right) g_{1}\left(t, x_{n}\right)
$$

for some germ $g_{1} \in C_{M^{s}}\left(\mathbf{R}^{2}, 0\right)$. It is now possible to argue just as in $[\mathbf{1 5}$, Subsection 3.3] to derive the inequality $s \geq \mu / m$, which, in view of (45), yields the desired conclusion.

4.4. Concluding remark. As mentioned in the introduction, the division problem for ultradifferentiable functions has been studied under another angle by Chaumat-Chollet [7]. Roughly speaking, they are able, by means of desingularization, to show that the quotient $f / \varphi$ enjoys $C_{M^{+}}$regularity with $M_{\ell}^{+}=M_{\ell}^{T}$ for some $T \geq 1$, not only in the case of two variables, but for higher dimensions as well. In counterpart, they have no control over the number $T$ and, in particular, nothing can be derived about the closedness of the ideal $I_{\varphi, M}$. Therefore, the viewpoint of [7] consists in working afterwards in a special subclass of $C_{M}$, say $\mathcal{C}$, defined as the intersection of all classes $C_{M^{\lambda}}$ for $\lambda>0$. The class $\mathcal{C}$ does not change when $M$ is replaced by $M^{T}$; thus, if $f$ belongs to $\mathcal{C}$, so does $f / \varphi$. In the same spirit, Bos-Milman [4] had previously considered some sort of "Gevrey estimates" under a fairly general, yet less precise, form. In contrast with these results, our approach gives optimal estimates for natural Carleman classes, but does not seem to work in the general higher-dimensional case. Also, we have to assume strong non-quasianalyticity since we use Borel type theorems, whereas [7] does not. It would be very interesting to get rid of both restrictions, especially regarding the dimension.

\section{References}

[1] E. Bierstone, Personal communication (1999).

[2] E. Bierstone and P.D. Milman, Semianalytic and subanalytic sets, Publ. Math. IHES, 67 (1988), 5-42, MR 89k:32011, Zbl 0674.32002.

[3] J. Bochnak and J.-J. Risler, Sur les exposants de Eojasiewicz, Comment. Math. Helvetici, 50 (1975), 493-507, MR 53 \#8474, Zbl 0321.32006.

[4] L. Bos and P.D. Milman, Sobolev-Gagliardo-Nirenberg and Markov type inequalities on subanalytic domains, Geom. and Funct. Anal., 5 (1995), 853-923, MR 97e:46038, Zbl 0848.46022 .

[5] J. Bruna, An extension theorem of Whitney type for non-quasianalytic classes of functions, J. London Math. Soc., 22 (1980), 495-505, MR 82e:58024, Zbl 0441.26015. 
[6] J. Chaumat and A.-M. Chollet, Caractérisation des anneaux nœtheriens de séries formelles à croissance contrôlée. Application à la synthèse spectrale, Publ. Mat., 41 (1997), 545-561, MR 98k:32010, Zbl 0906.46017.

[7] _ Sur la division et la composition dans des classes ultradifférentiables, Studia Math., 136 (1999), 49-70, MR 2000k:26033, Zbl 0942.26029.

[8] S. Izumi, A measure of integrity for local analytic algebras, Publ. RIMS, Kyoto University, 21 (1985), 719-735, MR 87i:32014, Zbl 0587.32016.

[9] H. Komatsu, Ultradistributions, I. Structure theorems and a characterization, J. Fac. Sci. Univ. Tokyo, Sect. IA, 20 (1973), 25-105, MR 47 \#9277, Zbl 0258.46039.

[10] S. Łojasiewicz, Sur le problème de la division, Studia Math., 18 (1959), 87-136, MR 21 \#5893, Zbl 0115.10203.

[11] B. Malgrange, Ideals of Differentiable Functions, Oxford University Press (1966), MR 35 \#3446, Zbl 0177.17902.

[12] M.A. Mostow and S. Shnider, Joint continuity of division of smooth functions I: Uniform Eojasiewicz estimates, Trans. Amer. Math. Soc., 292 (1985), 573-583, MR 87f:58018a, Zbl 0603.46034.

[13] H.-J. Petzsche, On E. Borel's theorem, Math. Ann., 282 (1988), 299-313, MR 89m:46076, Zbl 0633.46033.

[14] V. Thilliez, Sur les fonctions composées ultradifférentiables, J. Math. Pures et Appl., 76 (1997), 499-524, MR 99e:58018, Zbl 0878.58008.

[15] _ On closed ideals in smooth classes, Math. Nachr., 227 (2001), 143-157, MR 2002f:46101.

[16] Bounds for quotients in rings of formal power series with growth constraints, Studia Math., 151 (2002), 49-65.

[17] T. Wang, Linear Chevalley estimates, Trans. Amer. Math. Soc., 347 (1995), 48774897, MR 96k:32071, Zbl 0848.32002.

Received January 12, 2000

CNRS - UMR 8524

Mathématiques - BÂtiment M2

Université des Sciences et Technologies de Lille

59655 Villeneuve D'AscQ CedeX

FRANCE

E-mail address: Vincent.Thilliez@univ-lille1.fr 